\title{
Deformable Transparent All-Carbon-Nanotube
}

\section{Transistors}

Shinya Aikawa, ${ }^{1,2}$ Erik Einarsson, ${ }^{1,3}$ Theerapol Thurakitseree, ${ }^{1}$ Shohei Chiashi, ${ }^{1}$ Eiichi Nishikawa, ${ }^{2}$ and Shigeo Maruyama ${ }^{1, a)}$

${ }^{1}$ Department of Mechanical Engineering, The University of Tokyo, 7-3-1 Hongo, Bunkyo-ku, Tokyo 113-8656, Japan

${ }^{2}$ Department of Electrical Engineering, Tokyo University of Science, 1-3 Kagurazaka, Shinjuku-ku, Tokyo 162-8601, Japan

${ }^{3}$ Global Center of Excellence for Mechanical Systems Innovation, The University of Tokyo, 7-3-1 Hongo, Bunkyo-ku, Tokyo 113-8656, Japan

\begin{abstract}
:
We fabricated polymer-laminated, transparent, all-carbon-nanotube field-effect transistors (CNTFETs), making use of the flexible yet robust nature of single-walled carbon nanotubes (SWNTs). All components of the FET (active channel, electrodes, dielectric layer, and substrate) consist of carbon-based materials. The use of a plastic substrate that is considerably thinner than those used in other flexible CNT-FETs allowed our devices to be highly deformable without degradation of electrical properties. Using this approach, flexible, transparent CNT-FET devices able to withstand a $1 \mathrm{~mm}$ bending radius were realized.
\end{abstract}

a) Author to whom correspondence should be addressed. E-mail: maruyama@photon.t.u-tokyo.ac.jp 
Field-effect transistors (FETs) based on single-walled carbon nanotubes (SWNTs), ${ }^{1-12}$ single-crystal silicon, ${ }^{13}$ amorphous In-Ga-Zn-O (a-IGZO) ${ }^{14} \mathrm{In}_{2} \mathrm{O}_{3}$ nanowires, ${ }^{15}$ and organic materials ${ }^{16,17}$ are candidates for next-generation flexible and transparent electronic devices. However, flexible FETs typically use $\mathrm{Au}^{1-5,18-20}$ or ITO ${ }^{6,7,13-16}$ as the electrode material. The use of Au diminishes the optical transparency, whereas brittle ITO limits the mechanical flexibility of the device. ${ }^{21,22}$ To simultaneously improve upon both of these aspects, carbon nanotube FETs (CNT-FETs) employing SWNTs $^{8}$ or graphene $e^{9-12}$ for all electrodes (source, drain, and gate) have recently been reported. These metal-free devices can realize both optical transparency and mechanical robustness, but flexibility is typically sacrificed by fabricating the devices on thick plastic substrates. As suggested by Hur et al. ${ }^{2}$ and Cao et al., ${ }^{8}$ combining SWNTs with a thin, flexible material could lead to devices that are able to be wrinkled like paper. ${ }^{23}$

In this study, we demonstrate the realization of all-CNT-FETs in which all channels and electrodes (source, drain, and gate) are composed of SWNTs, and the substrate and dielectric layer are made of poly(vinyl alcohol) (PVA). The devices are fabricated using a simple transfer process followed by lamination using polymer sheets, and exhibit extreme flexibility and robustness to mechanical deformation.

A schematic illustrating the layered structure of the all-CNT-FET is shown in Fig. 1(a). To fabricate the device, a Si wafer was cut into 2 pieces, and on one of these pieces a 1 square inch active layer (S/D electrodes and channel) was prepared. On the other piece, a $0.5 \times 1$ inch global gate electrode was prepared. The larger substrate was patterned using standard photolithography processes (exposure and development). After patterning, Co catalyst (thickness: $0.5 \mathrm{~nm}$ ) was deposited onto both the photolithography-defined and non-patterned substrates using a thermal evaporator. The patterned substrate was then washed by dipping into acetone to remove residual resist (lift-off), rinsed with isopropyl alcohol (IPA), and dried using a nitrogen gun. SWNTs were then synthesized on both substrates using the alcohol catalytic chemical vapor deposition (ACCVD) 
method. ${ }^{24}$ Details regarding the synthesis procedure have been described elsewhere. ${ }^{25-27}$ This approach enables us to synthesize both the channels and S/D electrodes in a single CVD step. ${ }^{28}$

The device substrate and dielectric layers were prepared as follows. An aqueous polymer solution (concentration: 10\%) was prepared by dissolving PVA powder (Wako Pure Chemicals, degree of polymerization and saponification of 1500 and $86-90 \%$, respectively) into distilled water. The solution was spin coated (1500 rpm, $30 \mathrm{~s}$ ) over the SWNTs acting as the active layer and the global gate, and then dried at $65^{\circ} \mathrm{C}$ for $10 \mathrm{~min}$ on a hot plate. The global gate electrode was then peeled off from the substrate using tweezers. The gate electrode was stacked on top of the active layer after again spin coating $(3000 \mathrm{rpm}, 30 \mathrm{~s})$ and partial drying of the PVA solution. During the drying process $\left(65^{\circ} \mathrm{C}, 10 \mathrm{~min}\right)$ all three layers fused together, forming one integrated device. The entire device could then be peeled off from the Si master substrate. A schematic cross-section diagram of the FET is shown in Fig. 1(b). The final thickness of the device was approximately $15 \mu \mathrm{m}$.

Figure 1(c) shows a typical scanning electron microscope (SEM) image of the channel region of the all-CNT-FET with 5- $\mu \mathrm{m}$ channel length and width. The image was obtained prior to removal from the Si master substrate. The FET channel is formed by SWNTs that manage to bridge the gap between the source and drain electrodes. ${ }^{27}$ Figure 1(d) is a photograph of a functioning all-CNTFET device that has been heavily crumpled. The device is highly pliable because it is thinner than other commonly used transparent plastic supports such as poly(ethylene terephthalate) (PET) $)^{3,8,9}$ or poly(ethylene naphthalate) $(\mathrm{PEN}) .^{20,29}$

Using the fabrication method described above, the SWNT electrodes and channels were easily and completely transferred to a PVA film. Post-transfer SEM observations of the master substrate indicate no SWNTs remained after the transfer process (not shown). Kang et al. have reported ${ }^{30}$ that a carrier film such as $\mathrm{Au}$, as well as an intermediate layer between an adhesion polymer and target SWNTs, is necessary to remove SWNTs from growth substrate of crystal quartz. However, we have repeatedly found that PVA alone is sufficient to remove $\mathrm{SWNTs}$ from the $\mathrm{SiO}_{2} / \mathrm{Si}$ substrate. This is likely due to the substrate-nanotube interaction energy for amorphous $\mathrm{SiO}_{2}$ being considerably 
weaker than for crystal quartz. ${ }^{32}$ This weaker interaction results in random orientation of the SWNTs but makes it possible to remove the SWNTs from $\mathrm{SiO}_{2} / \mathrm{Si}$ using only PVA.

Figure 2(a) shows optical transmission spectra in the visible range for the PVA film and the contact pad (PVA/SWNT film) region. Optical transmittance of more than $80 \%$ was obtained, which is sufficient to see clearly through the device, as shown by the photograph of the device in Fig. 2(b).

Figure 3(a) is typical transfer characteristics for the FETs in flat, bent $(R=3.5 \mathrm{~mm}$ and $1.0 \mathrm{~mm})$, and wrinkled morphologies. The devices were characterized using a semiconductor parameter analyzer (Agilent 4156C) at room temperature under ambient conditions. An explanation for the ambipolar behavior of the PVA-laminated all-CNT-FETs will be reported elsewhere. ${ }^{33}$ Importantly, almost no changes in electrical properties were observed even though the devices were heavily deformed. To our knowledge, such a flexible yet robust SWNT-based device has not yet been realized. The main reason for the stable properties may be due to the robustness of the SWNTs themselves. ${ }^{34}$ However, the drain current $\left(I_{\mathrm{D}}\right)$ of a flexible FET using a SWNT-channel has been shown to degrade under mechanical strain or bending stress. ${ }^{1,3,2,29}$ In our device, the polymer substrate is approximately $1 / 10$ the thickness of other devices, thus the bending stresses on the channel is considerably reduced. Considering the Poisson's ratio of PVA, the change in axial strain should be small with respect to the transverse strain. Our measurements, however, show a slight increase in off current $\left(I_{\mathrm{OFF}}\right)$ when bent with a $1.0 \mathrm{~mm}$ radius of curvature (transverse strain: $0.75 \%$ ). The maximum $I_{\mathrm{D}}$, however, remained effectively unchanged. We suppose this behavior of $I_{\mathrm{OFF}}$ is due to the effective gate dielectric thickness, which changes with severe bending of the polymer sheet. The increased leak current recovers to the original level by returning the film shape to flat.

In order to investigate the mechanical stress tolerance of the all-CNT-FETs, 100 wrinkling cycles were performed at $V_{\mathrm{DS}}=-3 \mathrm{~V}$. The change in maximum drain current $\left(I_{\mathrm{D}, \max }\right)$ and the transconductance $\left(g_{\mathrm{m}}\right)$ as a function of the wrinkle cycles is shown in Fig. 3(b). A slight decrease in 
$I_{\mathrm{D}, \max }$ is observed. Due to the weak Van der Waals interaction between SWNTs, interconnections within the SWNT-network may be broken during deformation. However, $I_{\mathrm{D}}$ becomes stable against stresses after approximately 30 wrinkling cycles. The transconductance, in contrast, does not depend on the number of cycles. This implies that PVA is a sufficiently robust material and is very well suited for use as a flexible polymer dielectric.

To estimate the bendability of our all-CNT-FETs, we compared to other flexible transistors using SWNTs, ${ }^{3,8,11,29}$ amorphous In-Ga-Zn-O (a-IGZO), ${ }^{14}$ amorphous $\mathrm{Si}(a-\mathrm{Si}),{ }^{35}$ and pentacene $\mathrm{e}^{23,36,37}$ as the active channel. Since the bending parameters in references 3 and 8 are represented as strain $(\varepsilon)$ instead of radius of curvature $(R)$, we converted the strain values using the relation $\varepsilon=t_{\mathrm{s}} / 2 R$, where $t_{\mathrm{s}}$ is the substrate thickness. As shown in Fig. 4, our devices are the most bendable among flexible CNT-FETs, achieving $R=1.0 \mathrm{~mm}$ without $I_{\mathrm{D}}$ degradation. We expect our all-CNT-FETs could become even more flexible if the device structure were optimized to place the channels in a neutral position. $^{23,37}$

In conclusion, we realized all-CNT-FETs consisting only of SWNTs and polymer. The devices were fabricated by lamination of PVA/SWNT films, which included a simple PVA-only transfer process. The all-CNT-FETs had an optical transmittance of more than $80 \%$ in the visible region and were extremely flexible, able to withstand a bending radius of $1.0 \mathrm{~mm}$ without degradation in device performance. This work represents an important step toward realizing flexible and transparent all-carbon electronics.

\section{ACKNOWLEDGMENT}

Part of this work was financially supported by Grants-in-Aid for Scientific Research (19054003 and 22226006) and the Global COE Program 'Global Center for Excellence for Mechanical Systems Innovation'. One of the authors (SA) was financially supported by a JSPS 
Fellowship (21-1436). This work was also supported by the VLSI Design and Education Center (VDEC), The University of Tokyo, in collaboration with Cadence Corporation. 


\section{REFERENCES}

${ }^{1}$ E. Artukovic, M. Kaempgen, D. S. Hecht, S. Roth, and G. Grüner, Nano Lett. 5, 757 (2005).

${ }^{2}$ S.-H. Hur, O. O. Park, and J. A. Rogers, Appl. Phys. Lett. 86, 243502 (2005).

${ }^{3}$ Q. Cao, M.-G. Xia, M. Shim, and J. A. Rogers, Adv. Funct. Mater. 16, 2355 (2006).

${ }^{4}$ S. H. Tseng, and N. H. Tai, Appl. Phys. Lett. 95, 204104 (2009).

${ }^{5}$ T. Takenobu, N. Miura, S.-Y. Lu, H. Okimoto, T. Asano, M. Shiraishi, and Y. Iwasa, Appl. Phys. Express 2, 025005 (2009).

${ }^{6}$ F. N. Ishikawa, H. Chang, K. Ryu, P. Chen, A. Badmaev, L. G. De Arco, G. Shen, and C. Zhou, ACS Nano 3, 73 (2009).

${ }^{7}$ S. Kim, S. Ju, J. H. Back, Y. Xuan, P. D. Ye, M. Shim, D. B. Janes, and S. Mohammadi, Adv. Mater. 21, 564 (2009).

${ }^{8}$ Q. Cao, S.-H. Hur, Z.-T. Zhu, Y. G. Sun, C.-J. Wang, M. A. Meitl, M. Shim, and J. A. Rogers, Adv. Mater. 18, 304 (2006).

${ }^{9}$ S. Jang, H. Jang, Y. Lee, D. Suh, S. Baik, B. H. Hong, and J.-H. Ahn, Nanotechnology 21, 425201 (2010).

${ }^{10}$ B. Li, X. Cao, H. G. Ong, J. W. Cheah, X. Zhou, Z. Yin, H. Li, J. Wang, F. Boey, W. Huang, and H. Zhang, Adv. Mater. 22, 3058 (2010).

${ }^{11}$ W. J. Yu, S. Y. Lee, S. H. Chae, D. Perello, G. H. Han, M. Yun, and Y. H. Lee, Nano Lett. 11, 1344 (2011).

${ }^{12}$ W. J. Yu, S. H. Chae, S. Y. Lee, D. L. Duong, and Y. H. Lee, Adv. Mater. 23, 1889 (2011).

${ }^{13}$ E. Menard, R. G. Nuzzo, and J. A. Rogers, Appl. Phys. Lett. 86, 093507 (2005). 
${ }^{14}$ K. Nomura, H. Ohta, A. Takagi, T. Kamiya, M. Hirano, and H. Hosono, Nature 432, 488 (2004).

${ }^{15}$ S. Ju, A. Facchetti, Y. Xuan, J. Liu, F. Ishikawa, P. Ye, C. Zhou, T. J. Marks, and D. B. Janes, Nat. Nanotechnol. 2, 378 (2007).

${ }^{16}$ S.-J. Kim, J.-M. Song, and J.-S. Lee, J. Mater. Chem. 21, 14516 (2011).

${ }^{17}$ L. Basiricò, P. Cosseddu, B. Fraboni, and A. Bonfiglio, Thin Solid Films (Available online 5 May 2011) doi: 10.1016/j.tsf.2011.04.188.

${ }^{18}$ K. Bradley, J.-C. P. Gabriel, and G. Grüner, Nano Lett. 3, 1353 (2003).

${ }^{19}$ Q. Cao, H. Kim, N. Pimparkar, J. P. Kulkarni, C. Wang, M. Shim, K. Roy, M. A. Alam, and J. A. Rogers, Nature 454, 495 (2008).

${ }^{20}$ D. Sun, M. Y. Timmermans, Y. Tian, A. G. Nasibulin, E. I. Kauppinen, S. Kishimoto, T. Mizutani, and Y. Ohno, Nat. Nanotechnol. 6, 156 (2011).

${ }^{21}$ N. Saran, K. Parikh, D.-S. Suh, E. Muñoz, H. Kolla, and S. K. Manohar, J. Am. Chem. Soc. 126, $4462(2004)$.

${ }^{22}$ Y. Leterrier, L. Médico, F. Demarco, J.-A. E. Månson, U. Betz, M. F. Escolà, M. K. Olsson, and F. Atamny, Thin Solid Films 460, 156 (2004).

${ }^{23}$ T. Sekitani, U. Zschieschang, H. Klauk, and T. Someya, Nat. Mater. 9, 1015 (2010).

${ }^{24}$ S. Maruyama, R. Kojima, Y. Miyauchi, S. Chiashi, and M. Kohno, Chem. Phys. Lett. 360, 229 (2002).

${ }^{25}$ Y. Murakami, S. Chiashi, Y. Miyauchi, M. H. Hu, M. Ogura, T. Okubo, and S. Maruyama, Chem. Phys. Lett. 385, 298 (2004).

${ }^{26}$ S. Maruyama, E. Einarsson, Y. Murakami, and T. Edamura, Chem. Phys. Lett. 403, 320 (2005). 
${ }^{27}$ S. Aikawa, E. Einarsson, T. Inoue, R. Xiang, S. Chiashi, J. Shiomi, E. Nishikawa, and S. Maruyama, Jpn. J. Appl. Phys. 50, 04DN08 (2011).

${ }^{28}$ S. Aikawa, R. Xiang, E. Einarsson, S. Chiashi, J. Shiomi, E. Nishikawa, and S. Maruyama, Nano Res. 4, 580 (2011).

${ }^{29}$ T. Takenobu, T. Takahashi, T. Kanbara, K. Tsukagoshi, Y. Aoyagi, and Y. Iwasa, Appl. Phys. Lett. 88, 033511 (2006).

${ }^{30}$ S. J. Kang, C. Kocabas, H.-S. Kim, Q. Cao, M. A. Meitl, D.-Y. Khang, and J. A. Rogers, Nano Lett. 7, 3343 (2007).

${ }^{31}$ C. Kocabas, S. J. Kang, T. Ozel, M. Shim, and J. A. Rogers, J. Phys. Chem. C 111, 17879 (2007).

${ }^{32}$ J. Xiao, S. Dunham, P. Liu, Y. Zhang, C. Kocabas, L. Moh, Y. Huang, K.-C. Hwang, C. Lu, W. Huang, and J. A. Rogers, Nano Lett. 9, 4311 (2009).

${ }^{33} \mathrm{We}$ believe at present that the ambipolar behavior is attributed to the PVA layer possibly acting as a potential barrier, which suppresses electron transfer. However, further studies are underway to clarify the mechanism.

${ }^{34}$ D. Bozovic, M. Bockrath, J. Hafner, C. Lieber, H. Park, and M. Tinkham, Phys. Rev. B 67, 033407 (2003).

${ }^{35}$ L. Han, K. Song, P. Mandlik, and S. Wagner, Appl. Phys. Lett. 96, 042111 (2010).

${ }^{36}$ T. Sekitani, Y. Kato, S. Iba, H. Shinaoka, T. Someya, T. Sakurai, and S. Takagi, Appl. Phys. Lett. 86, 073511 (2005).

${ }^{37}$ T. Sekitani, S. Iba, Y. Kato, Y. Noguchi, T. Someya, and T. Sakurai, Appl. Phys. Lett. 87, 173502 (2005). 


\section{FIGURE CAPTIONS:}
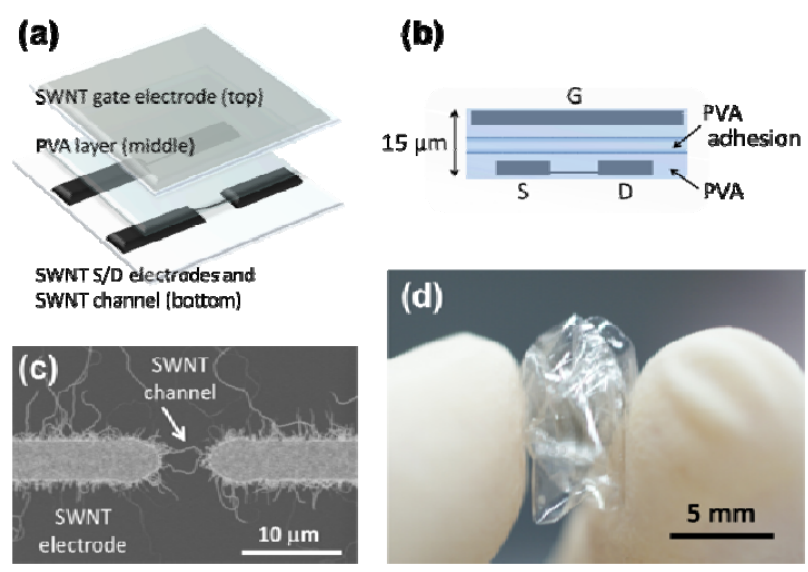

FIG. 1. (Color online) (a) Schematic of the layered structure of fabricated all-CNT-FETs. All electrodes (source, drain, and gate) and channels consist only of SWNTs. The substrate and dielectric layer are composed of flexible polymer. The top layer is the PVA-coated SWNT global gate electrode, the middle layer is the PVA adhesion layer acting as a dielectric, and the bottom layer contains PVA-coated SWNT source/drain electrodes and the SWNT channel. (b) Schematic cross-section diagram of the device. (c) SEM image of on the FET channel region obtained prior to removal from the master Si substrate. Both the channel length and width are $5 \mu \mathrm{m}$. (d) Photograph of a crumpled, yet functional, all-CNT-FET device.
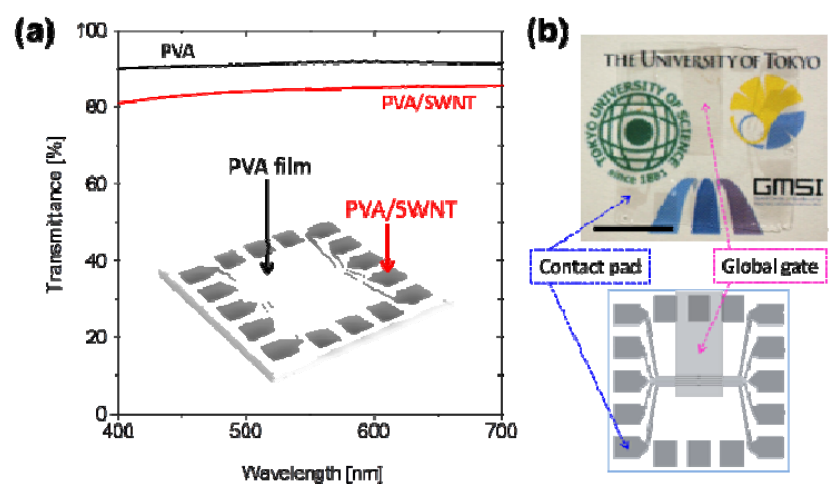

FIG. 2. (Color online) (a) Optical transmission spectra of the flexible all-CNT-FET in the visible range. Inset shows the locations where the spectra were obtained. (b) Optical image of the fabricated device in front of some printed logos (top) and the corresponding layout pattern (bottom). 
Scale bar: $1 \mathrm{~cm}$.
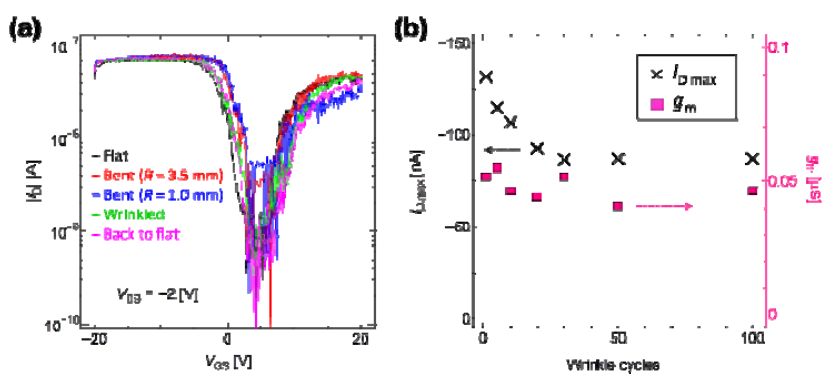

FIG. 3. (Color online) (a) Transfer characteristics of the flexible FET in flat, bent $(R=3.5 \mathrm{~mm})$, more bent $(R=1.0 \mathrm{~mm})$, and wrinkled. All $I-V$ curves were characterized at $V_{\mathrm{DS}}=-2 \mathrm{~V}$. (b) Mechanical stress tolerance values of the flexible all-CNT-FET over 100 wrinkle cycles. The black crosses and magenta squares indicate the maximum drain current $\left(I_{\mathrm{D} \max }\right)$ and the transconductance $\left(g_{\mathrm{m}}\right)$, respectively. The black dotted line is drawn to guide the eye.

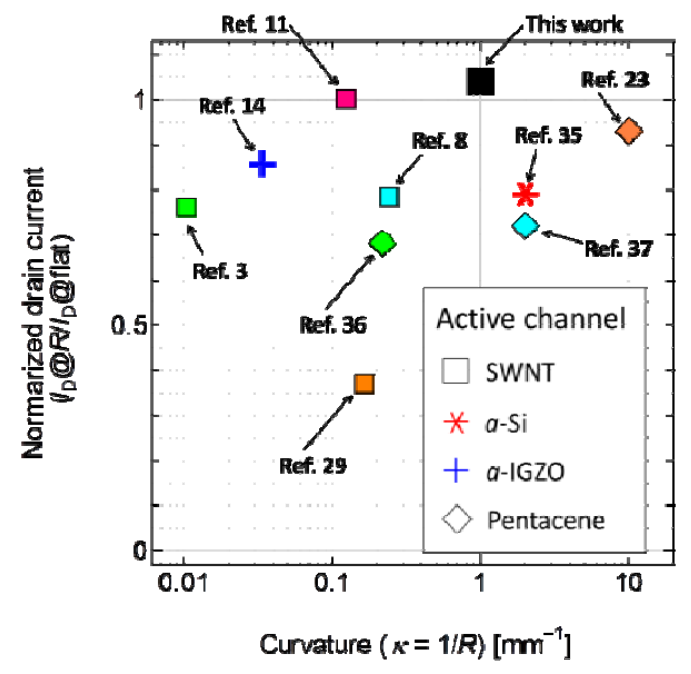

FIG. 4. (Color online) Comparison of curvature-dependent drain current $\left(I_{\mathrm{D}}\right)$ degradation between our all-CNT-FET and other flexible transistors based on SWNTs, amorphous In-Ga-Zn-O, amorphous $\mathrm{Si}$, and pentacene. The radius of curvature $(R)$ for refs 3 and 8 were calculated from reported strain values. 